\title{
Network-Coded Relaying in Multiuser Multicast D2D Network
}

\author{
Mingjun Dai, ${ }^{1,2,3}$ Shengli Zhang, ${ }^{1,2,3}$ Hui Wang, ${ }^{1,2,3}$ Bin Chen,,2,3 \\ Xiaohui Lin, ${ }^{1,2,3}$ Hongwei Liu, ${ }^{1,2,3}$ and Li Zhang ${ }^{1,2,3}$ \\ ${ }^{1}$ Shenzhen Key Lab of Advanced Communication and Information Processing, The College of Information Engineering, \\ Shenzhen University, Shenzhen 518060, China \\ ${ }^{2}$ The State Key Laboratory of Integrated Services Networks, Xidian University, Xian 710071, China \\ ${ }^{3}$ Shenzhen Key Laboratory of Media Security, Shenzhen University, Shenzhen 518060, China
}

Correspondence should be addressed to Shengli Zhang; zsl@szu.edu.cn

Received 25 June 2014; Revised 22 July 2014; Accepted 22 July 2014; Published 12 August 2014

Academic Editor: Lingyang Song

Copyright (C) 2014 Mingjun Dai et al. This is an open access article distributed under the Creative Commons Attribution License, which permits unrestricted use, distribution, and reproduction in any medium, provided the original work is properly cited.

D2D communication trades short-range communication for achieving high communication rate and short communication latency. Relay aided D2D communication can further tackle the problem of intermediate obstacles blocking the communication. In this work, multidevice multicast communication via a layer of parallel relay nodes is considered. Two relaying strategies, respectively, called the conventional relaying (CR) and network-coded relaying (NCR), are proposed. The throughput of these two schemes is analytically derived and evaluated through numerical study. Theoretically, NCR shows advantage over CR in twofold: one is higher throughput and the other is requiring less relay nodes and, hence, consuming less aggregate power. Numerical studies verify the analysis and show that the throughput performance gap between the two schemes increases significantly, actually exponentially with the number of devices.

\section{Introduction}

The main idea of D2D communication is to use short-range communication to trade for high rate communication, short delivery latency, and low aggregate consumption power [13]. Sometimes, due to the mobility of devices (devices move out of the communication coverage) or due to the fact that intermediate obstacles are blocking the communication, the communication link may be down intermittently [4]. These facts render relay-aided D2D communication necessary.

For relay-aided communication networks $[5,6]$, there are mainly two categories of strategies adopted by intermediate relay, including physical-layer relaying and network-coded relaying. For simple topology, for example, the point-to-point D2D communication via intermediate relays [7], capacity is given by the physical-layer technique in certain scenarios [810]. However, for complex topology, physical-layer technique tends to be unwieldy, while network coding (NC) [11, 12] shows significant advantages. Typical topology examples include interference network [13], multicast multihop network [14], intracell uplink relay network [15], and multiple unicasts networks [16] including three-node Alice-and-Bob relay network, two unicasts X-topology, cross topology, and wheel topology [17-25].

The full-duplex relay assumption in the above investigations is not practical [26]. In practice, if "cheap" relay is adopted, namely, half-duplex relay [27], which cannot transmit and receive simultaneously, the advantage of NC shown in the above investigations may be lost due to the orthogonal transmission nature of the multiple transmitters.

In this paper, we investigate a more general and practical model by considering the scenario where multiple devices exchange information via a layer of intermediate relays, namely, relay-aided D2D multicasting communication. For this model, we propose two relaying protocols based on the physical-layer and NC technique, respectively. Details of each technique are illustrated below.

Physical-Layer Technique. Firstly, for the special case where there is a single relay, the communication stage when all the devices transmit simultaneously can be viewed as multiple access channel (MAC) [28]. After this MAC stage, the 
relay broadcasts superimposed signals originating from the devices, and the corresponding channel can be viewed as broadcast channel (BC). In the BC stage, power allocation among all the information streams originating from different devices may degrade the system performance. In addition, due to heavy burden on the single relay, multiple cheap relays cooperately share the burden become a preference. One natural solution is that a lot of parallel relays are deployed between the devices, with each relay serving a single device, respectively, a device, a relay that serves it, and the other destination devices form a multicast single relay network. We call this strategy conventional multicast relaying (CMCR). The most desirable feature of this scheme is the simple operation at each relay. However, since each relay only decodes one device's information while treating the other devices' signals as interference, the performance of CMCR is interference-limited [29].

NC Technique. After the MAC stage, each relay firstly applies $\mathrm{NC}$ operation on some information flows originating from different devices and then multicasts the resultant information flow to all the devices. Each device can employ the idea of side information-aided decoding [30]. More specifically, each device performs decoding utilizing the message originating from itself. We call this strategy network-coded multicast relaying (NCMCR). The most desirable feature of NCMCR is that the interference can be reduced to some extent, since more devices' signals are decoded at each relay and hence less devices' signals are treated as interference. Besides, this does not involve power allocation among the information streams at each relay node, which also embodies NC's advantage over the physical relaying technique.

\section{System Model}

Refer to Figure 1. Consider $M$ devices, denoted as $U_{i}$ for $i=$ $\{1,2, \ldots, M\} \triangleq \mathscr{M}$, exchanging information over a layer of $N$ parallel relays, denoted as $R_{i}$ for $i=\{1,2, \ldots, N\} \triangleq \mathscr{N}$. Define device terminal set $\mathcal{U} \triangleq\left\{U_{1}, U_{2}, \ldots, U_{M}\right\}$ and relay node set $\mathscr{R} \triangleq\left\{R_{1}, R_{2}, \ldots, R_{N}\right\}$. Device $U_{i}$ multicasts information $m_{i}$ to the other devices $\mathscr{U} /\left\{U_{i}\right\}, i \in \mathscr{M}$. The devices are assumed to be so far away that the wireless link between them can be neglected. We assume that the communication between any pair of devices experiences two hops, that is, through certain relay nodes. Each node in the network is assumed to have one single antenna and operates in half-duplex mode. Node $i \in \mathscr{U} \cup \mathscr{R}$ is subject to power constraint $P_{i}$. For simplicity, we consider additive white Gaussian noise (AWGN) at the receiver.

The communication between any pair of devices is performed in two time slots. During the first time slot, the devices simultaneously distribute their data while the relays listen. During the second time slot, the relays forward the messages to the devices.

Let $X_{i}[m], Y_{i}[m]$, and $w_{i}[m]$ be, respectively, the transmitted symbol from node $i$, the received symbol, and the thermal noise at node $i$, at time $m$, respectively, $i \in \mathscr{U} \cup \mathscr{R}$.



FIGURE 1: The general model.

The first hop's input-output relationship is represented by the following formula:

$$
Y_{R_{i}}[m]=\sum_{j \in \mathscr{U}} X_{U_{j}}[m]+w_{i}[m], \quad i \in \mathcal{N}, j \in \mathscr{M},
$$

which is subject to

$$
\operatorname{Var}\left\{X_{U_{j}}[m]\right\} \leq P_{U_{j}}
$$

For simplicity, we assume that $P_{i}=P$ and $w_{i}[m] \sim \mathscr{C} \mathcal{N}\left(0, \sigma^{2}\right)$ for all $i \in \mathscr{U} \cup \mathscr{R}$. Define signal-to-noise ratio (SNR) $\Gamma \triangleq P / \sigma^{2}$. The second hop's input-output relationship depends on the selected transmission scheme and is hence detailed in the following sections. Define $R_{m_{i}}$ and $R_{m_{i}, m_{j}}$ as the data rate of information flow originating from source $U_{i}$ and the data rate of information flows originating from sources $U_{i}$ and $U_{j}$, respectively, $i, j \in \mathcal{U}, i \neq j$.

Remark 1. Throughout this paper, we assume that matchedfilter receiver is used; hence, the achievable rate in each hop can be denoted by its capacity, and we let $C(x) \triangleq \log _{2}(1+x)$.

\section{Proposed Transmission Protocols}

For easy understanding, we first consider the special case where there are three devices in Section 3.1. We then generalize the above results to an arbitrary number of devices together with mathematical analysis, that is, to $M>3$, in Section 3.2.

3.1. Proposed Schemes for Three Devices. We describe the conventional method, the proposed method, and the refined proposed method, respectively. 


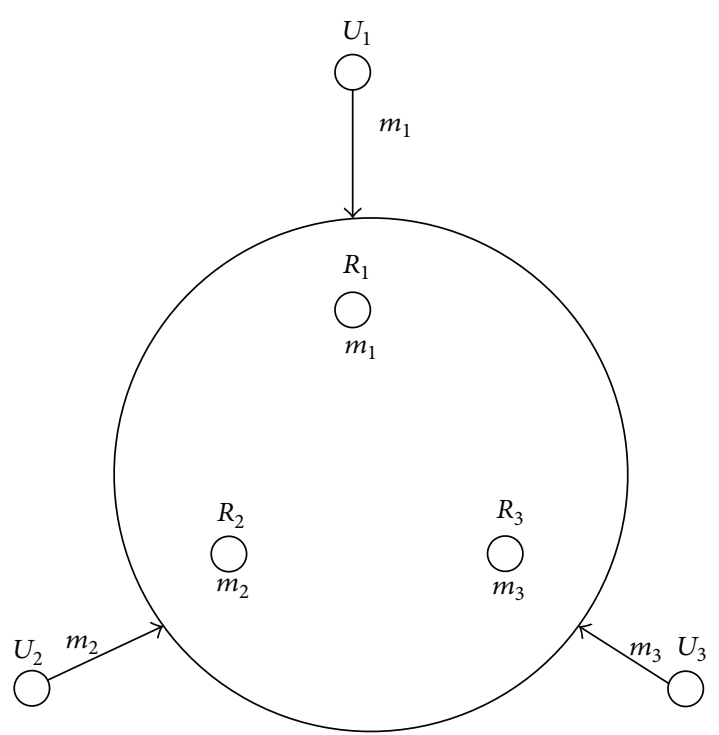

Figure 2: Conventional relaying.

3.1.1. Conventional Multicast Relaying (CMCR). Refer to Figure 2. Relay $R_{i}$ adopts decode-and-forward (DF) strategy to relay device $U_{i}$ 's signal to the other devices, $i \in\{1,2,3\} \triangleq$ $\mathcal{U}_{3}$. In the following, we derive the achievable rate of each device. Note that there are totally two stages of transmission and the achievable rate is the minimum of these two stages.

In the first stage, since relay $R_{i}$ only decodes the message originating from device $U_{i}$, the signals originating from other devices' are treated as interference, $i \in \mathcal{U}_{3}$. In this case, the achievable rate region is

$$
R_{m_{i}} \leq C\left(\frac{P_{U_{i}}}{\sigma^{2}+\sum_{j \in U_{3}, j \neq i} P_{U_{j}}}\right),
$$

which is simplified to

$$
R_{m_{i}} \leq C\left(\frac{1}{1 / \Gamma+2}\right)
$$

In the second stage, device $U_{i}$ intends to decode the messages from relays $R_{j}$ 's for $j \in \mathscr{U}_{3} /\{i\}$. This stage can be viewed as a two-user MAC by treating the signal from relay $R_{i}$ as interference. In this case, the achievable rate region is

$$
\begin{gathered}
R_{m_{j}} \leq C\left(\frac{P_{R_{j}}}{\sigma^{2}+P_{R_{i}}}\right), \quad j \in \mathscr{U}_{3} /\{i\}, \\
R_{m_{j}}+R_{m_{k}} \leq C\left(\frac{P_{R_{j}}+P_{R_{k}}}{\sigma^{2}+P_{R_{i}}}\right), \quad j, k \in \mathcal{U}_{3} /\{i\}, j \neq k .
\end{gathered}
$$

From (5), we obtain that the achievable rate region of the message rate originating from device $i$ in the second stage is

$$
R_{m_{i}} \leq \frac{1}{2} C\left(\frac{2}{1 / \Gamma+1}\right)
$$



FIgURE 3: Network-coded relaying.

Combining (4) and (6), we obtain that the achievable rate region of each device by CMCR is

$$
\begin{aligned}
R_{m_{i}}^{\mathrm{CMCR}} & \leq \min \left(C\left(\frac{1}{1 / \Gamma+2}\right), \frac{1}{2} C\left(\frac{2}{1 / \Gamma+1}\right)\right) \\
& =C\left(\frac{1}{1 / \Gamma+2}\right) .
\end{aligned}
$$

3.1.2. Network-Coded Multicast Relaying (NCMCR). Refer to Figure 3. Device $U_{i}$ selects relays $R_{j}$ for all $j \in \mathcal{U}_{3} \backslash\{i\}$ as the intermediate relays to forward its message to the other devices. Relay $R_{j}$ performs the following procedures. Firstly, it decodes the messages from devices $U_{i}$ for $i \in \mathscr{U}_{3} \backslash\{j\}$. Afterwards, it performs exclusive OR (XOR) operation on the decoded information bits. Finally, it encodes the resultant information bits into new codeword and sends the resultant codeword out. We derive the achieved rate region in the following.

In the first stage, relay $R_{i}$ needs to decode the signals from devices $U_{j}$ 's for $j \in \mathcal{U}_{3} \backslash\{i\}$. This stage can be viewed as a twouser MAC. The achievable rate region is

$$
\begin{gathered}
R_{m_{j}} \leq C\left(\frac{P_{U_{j}}}{\sigma^{2}+P_{U_{i}}}\right), \quad j \in \mathscr{U}_{3} /\{i\}, \\
R_{m_{j}}+R_{m_{k}} \leq C\left(\frac{P_{U_{j}}+P_{U_{k}}}{\sigma^{2}+P_{U_{i}}}\right), \quad j, k \in \mathscr{U}_{3} /\{i\}, j \neq k .
\end{gathered}
$$

From (9), we obtain the achievable rate region of device $U_{i}$ in the first stage as

$$
R_{m_{i}} \leq \frac{1}{2} C\left(\frac{2}{1 / \Gamma+1}\right)
$$

In the second stage, since device $U_{i}$ intends to decode the messages from relays $R_{j}$ 's for $j \in \mathcal{U}_{3} \backslash\{i\}$, the channel can also 
be viewed as a two-user MAC. The achievable rate region at decoder $U_{i}$ is

$$
\begin{gathered}
R_{m_{i}, m_{j}} \leq C\left(\frac{P_{R_{j}}}{\sigma^{2}+P_{R_{i}}}\right), \quad j \in \mathcal{U}_{3} \backslash\{i\}, \\
R_{m_{i}, m_{j}}+R_{m_{i}, m_{k}} \leq C\left(\frac{P_{R_{j}}+P_{R_{k}}}{\sigma^{2}+P_{R_{i}}}\right), \quad j, k \in \mathcal{U}_{3} \backslash\{i\}, j \neq k .
\end{gathered}
$$

Note that

$$
R_{m_{i}, m_{j}}=\min \left(R_{m_{i}}, R_{m_{j}}\right), \quad i, j \in \mathcal{U}_{3}, i \neq j
$$

From (11) and (12), we obtain that the achievable rate region of device $U_{i}$ in the second stage is

$$
R_{m_{i}} \leq \frac{1}{2} C\left(\frac{2}{1 / \Gamma+1}\right) .
$$

Combining (10) and (13), we obtain that the achievable rate region of device $U_{i}$ by NCMCR is

$$
R_{m_{i}}^{\mathrm{NCMCR}} \leq \frac{1}{2} C\left(\frac{2}{1 / \Gamma+1}\right)
$$

3.1.3. Refined NCMCR. Combining (8) and (14), we can obtain

$$
R_{m_{i}}^{\mathrm{NCMCR}}>R_{m_{i}}^{\mathrm{CMCR}}
$$

which shows the advantage of NCMCR over CMCR. However, for more than 3 devices, the number of relays needed by intuitively applying NCMCR should be $C_{M}^{2}$, while CMCR requires only $M$ relays.

In the following, we propose a method based on NCMCR to reduce the number of relays required to be even less than the number of devices. Without loss of generality, we first illustrate the case for $M=3$. We remove relay $R_{1}$ and keep the operations performed on the other relay nodes the same as those described in Section 3.1.2 (refer to Figure 4). Obviously, the decoding at device $U_{1}$ can be made the same as before. Now, let us consider the decoding operation at devices $U_{2}$ and $U_{3}$. Without loss of generality, we consider node $U_{2}$ only. It intends to decode $m_{1}$ and $m_{3}$. Since $m_{2}$ is available to $U_{2}$ and hence can be viewed as side information [31], node $U_{2}$ first performs XOR operation on $m_{2}$ with $m_{1} \oplus m_{2}$ which is forwarded by relay $R_{2}$, obtaining $m_{1}$. Afterwards, $U_{2}$ performs XOR operation again on $m_{1}$ and $m_{1} \oplus m_{3}$ which is forwarded by relay $R_{3}$, obtaining $m_{3}$. We name this protocol as refined network-coded multicast relaying (RNCMCR). In the following, we derive the achievable rate region of RNCMCR.

In the first stage, consider relay $R_{2}$. It needs to decode the message from devices $U_{1}$ and $U_{2}$, respectively. The channel



FIGURE 4: Two relays.

can be viewed as a two-user MAC. The achievable rate region is, therefore,

$$
\begin{gathered}
R_{m_{j}} \leq C\left(\frac{P_{U_{j}}}{\sigma^{2}+P_{U_{3}}}\right), \quad j \in\{1,2\}, \\
R_{m_{1}}+R_{m_{2}} \leq C\left(\frac{P_{U_{1}}+P_{U_{2}}}{\sigma^{2}+P_{U_{3}}}\right) .
\end{gathered}
$$

Similarly, for relay $R_{3}$ in the first stage, we have

$$
\begin{gathered}
R_{m_{j}} \leq C\left(\frac{P_{U_{j}}}{\sigma^{2}+P_{U_{2}}}\right), \quad j \in\{1,3\}, \\
R_{m_{1}}+R_{m_{3}} \leq C\left(\frac{P_{U_{1}}+P_{U_{3}}}{\sigma^{2}+P_{U_{2}}}\right) .
\end{gathered}
$$

We then obtain that the achievable rate region of device $U_{i}$ in the first stage is

$$
R_{m_{i}} \leq \frac{1}{2} C\left(\frac{2}{1 / \Gamma+1}\right) .
$$

In the second stage, for device $U_{2}$, we have

$$
\begin{aligned}
& R_{m_{1}, m_{2}} \leq C\left(\frac{P_{R_{2}}}{\sigma^{2}}\right)=C(\Gamma), \\
& R_{m_{1}, m_{3}} \leq C\left(\frac{P_{R_{3}}}{\sigma^{2}}\right)=C(\Gamma), \\
& R_{m_{1}, m_{2}}+R_{m_{1}, m_{3}} \leq C\left(\frac{P_{R_{2}}+P_{R_{3}}}{\sigma^{2}}\right)=C(2 \Gamma) .
\end{aligned}
$$

We then obtain that the achievable rate region of device $U_{i}$ in the second stage is

$$
R_{m_{i}} \leq \frac{1}{2} C(2 \Gamma)
$$


Combining (18) with (20), we obtain that the achievable rate region of device $U_{i}$ by RNCMCR is

$$
R_{m_{i}}^{\mathrm{RNCMCR}} \leq \frac{1}{2} C\left(\frac{2}{1 / \Gamma+1}\right),
$$

which is the same as (14). It naturally indicates a conjecture that, for $M$ devices, $M-1$ relays are enough for RNCMCR without performance penalty with respect to that composed of $C_{M}^{2}$ relays. In the next subsection, we analytically verify this conjecture and compare RNCMCR with CMCR.

3.2. Analysis for General Number of Devices. We first derive the achievable rate region obtained by CMCR and RNCMCR, respectively. We then compare these two schemes.

3.2.1. CMCR. Following the above derivation steps, we can obtain that the achievable rate region by CMCR for $M$ devices and $M$ relays in each stage is as follows.

Stage 1. Consider

$$
R \leq C\left(\frac{1}{t+M-1}\right)
$$

where $t \triangleq 1 / \Gamma$.

Stage 2. Consider

$$
R \leq \frac{1}{M-1} C\left(\frac{M-1}{t+1}\right) .
$$

By jointly considering these two stages, we obtain

$$
\begin{aligned}
R^{\mathrm{CMCR}} & \leq \min \left(C\left(\frac{1}{t+M-1}\right), \frac{1}{M-1} C\left(\frac{M-1}{t+1}\right)\right) \\
& =C\left(\frac{1}{t+M-1}\right),
\end{aligned}
$$

where the proof of (25) is given in The Appendix.

3.2.2. RNCMCR. Following the above derivation steps, we can obtain that the achievable rate region by RNCMCR for $M$ devices and $M-1$ relays in each stage as follows.

Stage 1. Consider

$$
R \leq \frac{1}{M-1} C\left(\frac{M-1}{t+1}\right) .
$$

Stage 2. Consider

$$
R \leq \frac{1}{M-1} C\left(\frac{M-1}{t}\right) .
$$

By jointly considering these two stages, we obtain

$$
\begin{aligned}
R^{\mathrm{RNCMCR}} & \leq \min \left(\frac{1}{M-1} C\left(\frac{M-1}{t+1}\right), \frac{1}{M-1} C\left(\frac{M-1}{t}\right)\right) \\
& =\frac{1}{M-1} C\left(\frac{M-1}{t+1}\right) .
\end{aligned}
$$



FIgURE 5: Throughput comparison at different SNRs, $M=3$.

3.2.3. Comparison. Comparing (25) with (29), we obtain

$$
R^{\mathrm{RNCMCR}}>R^{\mathrm{CMCR}} .
$$

It reveals the fact that RNCMCR outperforms CMCR twofold. On one hand, it uses less relays and hence needs less aggregate power budget. On the other hand, according to (30), RNCMCR outperforms CMCR in terms of achievable rate.

\section{Numerical Study}

In this section, we compare the achievable throughput of different schemes, where throughput is defined as the sum rate of all devices in the network. We set $\sigma=1$.

For $M=3$, the achievable throughput of NCMCR and CMCR is plotted in Figure 5. We can see that the performance improvement in the high SNR region is around 33\%.

In Figure 6, we consider the case where $M=100$. We can see that the performance improvement is much more significant than the $M=3$ case.

To show the advantage under different number of devices, we plot Figure 7 with the horizontal axis representing the number of devices. We can see that the performance advantage increases linearly with respect to the logarithm of $M$. This indicates that our proposed scheme scales well with the network size (in terms of the number of devices).

\section{Conclusion}

The throughput of a D2D communication aided by a multidevice multicast two-hop Gaussian parallel relay network is analyzed. Both conventional relaying (CR) strategy and network-coded relaying (NCR) strategy together with refined version are proposed. Their achievable rates are evaluated theoretically and numerically. Comparison results show that 




FIgURE 6: Throughput comparison at different SNRs, $M=100$.



Figure 7: Throughput comparison with respect to $M, \Gamma=50 \mathrm{~dB}$.

NCR outperforms CR; the advantage increases linearly with respect to the logarithm of the number of devices, which indicates that the proposed scheme scales well with the network size.

\section{Appendix}

\section{Proof for (25)}

Proof. Since the case where $M=2$ is just the Alice-and-Bob model which has been considered in [32] and the case where
$M=3$ has been analyzed previously, it suffices to prove for the cases $M \geq 4$ that the following equation holds:

$$
C\left(\frac{1}{t+M-1}\right)<\frac{1}{M-1} C\left(\frac{M-1}{t+1}\right) .
$$

It is equivalent to prove

$$
\begin{aligned}
1+ & \frac{1}{t+M-1}<\left(1+\frac{M-1}{t+1}\right)^{1 /(M-1)} \\
& \Longleftrightarrow\left(\frac{t+M}{t+M-1}\right)^{M-1}<\frac{t+M}{t+1} \\
& \Longleftrightarrow\left(\frac{t+M}{t+M-1}\right)^{M-2}<\frac{t+M-1}{t+1} .
\end{aligned}
$$

At high SNR, we have $t \rightarrow 0$. Hence, (A.4) is equivalent to

$$
\begin{aligned}
& \left(\frac{M}{M-1}\right)^{M-2}<M-1 \\
& \Longleftrightarrow\left(1+\frac{1}{M-1}\right)^{M-2}<M-1 .
\end{aligned}
$$

Note that $f(x) \triangleq(1+(1 / x))^{x-1}$ monotonically increases with respect to $x$ when $x>0$ and $\lim _{x \rightarrow \infty} f(x)=e<3 \leq M-1$ when $M \geq 4$. We hence obtain (25).

\section{Conflict of Interests}

The authors declare that there is no conflict of interests regarding the publication of this paper.

\section{Acknowledgments}

This work was supported by Grants from Natural Science Foundation of China (61301182, 61372078, 60602066, 60773203 , and 61171071), from the National 973 project (2013CB336700), from Natural Science Foundation of Guangdong Province (S2013040016857), from Specialized Research Fund for the Doctoral Program of Higher Education from The Ministry of Education (20134408120004), from Yumiao Engineering from Education Department of Guangdong Province (2013LYM_0077), from the Open Research Fund of the State Key Laboratory of Integrated Services Networks, Xidian University (ISN15-06), from Foundation of Shenzhen City (JC201005280404A, JC201005250035A, JC201005250047A, JCYJ20120613115037732, JCYJ20120613174214967, ZDSY20120612094614154, GJHS20120621143440025, JCYJ20120613115442060, and C201005250085A), and from Natural Science Foundation of Shenzhen University (00036107).

\section{References}

[1] L. Song, D. Niyato, Z. Han, and E. Hossain, Wireless Device-toDevice Communications and Networks, Cambridge University Press, 2013.

[2] L. Song, D. Niyato, Z. Han, and E. Hossain, "Game-theoretic resource allocation methods for device-to-device communication," IEEE Wireless Communications, vol. 21, no. 3, pp. 136-144, 2014. 
[3] C. Xu, L. Song, D. Zhu, and M. Lei, "Subcarrier and power optimization for device-to-device underlay communication using auction games," in Proceedings of the IEEE International Conference on Communications (ICC), Sydney, Australia, June 2014.

[4] H. Cui, L. Song, and B. Jiao, "Multi-pair two-way amplify-andforward relayingwith very large number of relay antennas," IEEE Transactions on Wireless Communications, vol. 13, no. 5, pp. 2636-2645, 2014.

[5] L. Song, "Relay selection for two-way relaying with amplifyand-forward protocols," IEEE Transactions on Vehicular Technology, vol. 60, no. 4, pp. 1954-1959, 2011.

[6] F. Sun, E. de Carvalho, P. Popovki, and C. Thai, "Coordinated direct and relay transmission with linear non-regenerative relay beamforming," IEEE Transactions on Vehicular Technology, vol. 60, no. 4, pp. 1954-1959, 2011.

[7] L. Song, J. Shen, and S. Sun, "Research progress on device-todevice communication in China," IEEE COMSOC MMTC ELetter, vol. 9, no. 1, pp. 46-48, 2014.

[8] T. M. Cover and A. A. El Gamal, "Capacity theorems for the relay channel," Institute of Electrical and Electronics Engineers. Transactions on Information Theory, vol. 25, no. 5, pp. 572-584, 1979.

[9] A. Host-Madsen and J. Zhang, "Capacity bounds and power allocation for wireless relay channels," IEEE Transactions on Information Theory, vol. 51, no. 6, pp. 2020-2040, 2005.

[10] G. Kramer, M. Gastpar, and P. Gupta, "Cooperative strategies and capacity theorems for relay networks," IEEETransactions on Information Theory, vol. 51, no. 9, pp. 3037-3063, 2005.

[11] R. Ahlswede, N. Cai, S. R. Li, and R. W. Yeung, "Network information flow," Institute of Electrical and Electronics Engineers. Transactions on Information Theory, vol. 46, no. 4, pp. 12041216, 2000.

[12] L. Song, G. Hong, B. Jiao, and M. Debbah, "Joint relay selection and analog network coding using differential modulation in two-way relay channels," IEEE Transactions on Vehicular Technology, vol. 59, no. 6, pp. 2932-2939, 2010.

[13] S. Bhadra, P. Gupta, and S. Shakkottai, "On network coding for interference networks," in Proceedings of the IEEE International Symposium on Information Theory (ISIT '06), pp. 207-211, Seattle, Wash, USA, July 2006.

[14] Y. H. Kim, N. S. Seo, and Y. Y. Kim, "A network coding based multicasting (NETCOM) over IEEE 802.11 multi-hop," in Proceedings of the IEEE 65th Vehicular Technology Conference (VTC '07), pp. 845-848, Dublin, Ireland, April 2007.

[15] Y. Chen, S. Kishore, and J. Li, "Wireless diversity through network coding," in Proceeding of the IEEE Wireless Communications and Networking Conference (WCNC '06), vol. 3, pp. 16811686, Las Vegas, Nev, USA, April 2006.

[16] M. Dai, H. Y. Kwan, and C. W. Sung, "Linear network coding strategies for the multiple access relay channel with packet erasures," IEEE Transactions on Wireless Communications, vol. 12, no. 1, pp. 218-227, 2013.

[17] L. Song, Y. Li, A. Huang, B. Jiao, and A. V. Vasilakos, "Differential modulation for bidirectional relaying with analog network coding," IEEE Transactions on Signal Processing, vol. 58, no. 7, pp. 3933-3938, 2010.

[18] F. Sun, T. M. Kim, A. J. Paulraj, E. de Carvalho, and P. Popovski, "Cell-edge multi-user relaying with overhearing," IEEE Communications Letters, vol. 17, no. 6, pp. 1160-1163, 2013.
[19] C. Li, L. Yang, and W. Zhu, "Two-way MIMO relay precoder design with channel state information," IEEE Transactions on Communications, vol. 58, no. 12, pp. 3358-3363, 2010.

[20] S. Katti, H. Rahul, W. Hu, D. Katabi, M. Medard, and J. Crowcroft, "XORs in the air: practical wireless network coding," IEEE/ACM Transactions on Networking, vol. 16, no. 3, pp. 497510, 2008.

[21] C. Li, L. Yang, and Y. Shi, "An asymptotically optimal cooperative relay scheme for two-way relaying protocol," IEEE Signal Processing Letters, vol. 17, no. 2, pp. 145-148, 2010.

[22] S. Zhang, S. C. Liew, and P. P. Lam, "Hot topic: physical-layer network coding," in Proceedings of the 12th Annual International Conference on Mobile Computing and Networking (MOBICOM '06), pp. 358-365, Los Angeles, Calif, USA, September 2006.

[23] M. Effros, T. Ho, and S. Kim, "A tiling approach to network code design for wireless networks," in Proceedings of the Information Theory Workshop, Punta del Este, Uruguay, March 2006.

[24] B. Ghelber and R. Dabora, "The value of cooperation between relays in the multiple-access channel with multiple relays," European Transactions on Telecommunications, vol. 23, no. 4, pp. 341-359, 2012.

[25] M. Dai, H. Wang, X. Lin, S. Zhang, and B. Chen, “Opportunistic relaying with analog and digital network coding for two-way parallel relay channels," IET Communications, vol. 8, no. 12, pp. 2200-2206, 2014.

[26] M. Dai, P. Wang, S. Zhang et al., "Survey on cooperative strategies for wireless relay channels," Transactions on Emerging Telecommunications Technologies, 2014.

[27] P. Rost and G. Fettweis, "Analysis of decode-and-forward, compress-and-forward and combined protocols for multiterminal half-duplex relay networks with random schedules," European Transactions on Telecommunications, vol. 24, no. 2, pp. 196-211, 2013.

[28] P. Wang and L. Ping, "On maximum eigenmode beamforming and multi-user gain," IEEE Transactions on Information Theory, vol. 57, no. 7, pp. 4170-4186, 2011.

[29] P. Wang and L. Ping, "MIMO multiple access via maximum eigenmode beamforming," IEEE Transactions on Vehicular Technology, vol. 61, no. 2, pp. 900-906, 2012.

[30] T. M. Cover and J. A. Thomas, Elements of Information Theory, John Wiley \& Sons, New York, NY, USA, 2006.

[31] M. Dai, K. W. Shum, and C. W. Sung, "Data dissemination with side information and feedback," to appear in IEEE Transactions on Wireless Communications, 2014.

[32] P. Larsson, N. Johansson, and K.-E. Sunell, "Coded bidirectional relaying," in Proceedings of the 5th Scandianavian Workshop on Wireless Ad-Hoc Networks, Stockholm, Sweden, May 2005. 





The Scientific World Journal


Submit your manuscripts at http://www.hindawi.com



Journal of

Control Science

and Engineering
